\title{
SOWING THE SEEDS OF COLLABORATIVE CURIOSITY: FOSTERING ACADEMIC RESEARCH SKILLS IN PRE-BACHELOR CREATIVE ARTS STUDENTS
}

\author{
Daniel Wagner \\ Unitec Institute of Technology (Auckland, New Zealand)
}

\begin{abstract}
Conducting and presenting academic research is an acquired craft. Most students who enter higher education have no previous experience in the research domain, and many spend the first semester (or more) of their bachelor degree grappling with the nature and scope of this new challenge. Much of the course content in students' first semester is lost in the shuffle of coming to grips with tertiary study itself. To address this, efforts are underway to arm students with fundamental research skills before they begin their degree. In a pre-bachelor certificate programme at Unitec Institute of Technology in Auckland, New Zealand, creative arts students have been given a simple system to learn how to engage in and present academic research. Using a suite of online tools, the students collaborate to develop a team research question, perform the research, and present their findings and conclusions. The research process is demystified for them and they are empowered with new skills and knowledge around how to channel their natural curiosity into an academic framework. Along the way, they also develop presentation skills as well as becoming familiar with using tools for remote collaboration. This paper will discuss the students' journey through the course and will share insight into the value to students of beginning a bachelor degree pre-armed with basic research skills.
\end{abstract}

\section{INTRODUCTION}

The literature is bountiful in support of the development of academic research skills as part of the undergraduate experience. Lanza (1988) extolls the value of undergraduate research for its ability to strengthen verbal and written communication skills as well as providing the student with "an emotional experience that is beneficial in subsequent enterprises" [1]. Lopatto (2010) cites skills gains reported by students in a number of disciplines resulting from undergraduate research, as well as the enhancement of knowledge around research design and information literacy. He goes on to assert that "many educators... have come to see the potential for... academic research to be a high-impact educational practice for achieving excellence in liberal education." [2] Bowers \& Parameswaran (2013) suggest that the integration of research into undergraduate courses "would significantly improve both the undergraduate experience generally and broader educational outcomes" [3].

Indeed, several programmes have been developed and adopted over the years to nurture undergraduate research skills, including the CLIMB project [4] and the SURE project [2]. But at our busy performing and screen arts school, where students' timetables runneth over with acting and film projects, there is not much bandwidth left for the theory courses that are woven into each semester. Academic staff have identified that even a very basic introduction to research (and presentation) skills before entering the degree would set the students up for success by acclimating them to the research mindset.

This, then, is a report about one such effort to offer pre-degree students a small taster of the research experience. I will begin with a description of the Bachelor degree for which the Certificate prepares the students.

\section{CONTEXT}

\subsection{The Bachelor Degree}

The Bachelor of Performing and Screen Arts (BPSA), which sits within the School of Creative Industries at Unitec Institute of Technology, provides technical and theoretical training by industry professionals and offers endorsements (majors) in Contemporary Dance, Acting, Performance Technology and Screen Arts (which is the film school). Teaching in the BPSA's four majors is centred around performances or projects of various sizes. There is constant "making" in the BPSA.

The degree also has a theoretical component wherein each semester, students take courses such as "Storytelling, Myth, and Ritual", "Genre, Time, and Meaning", and "Audience, Context, and Interpretation". Here, they engage academically with the creative works of those who have come 
before them and with the intricate interrelationships between creativity and culture.

The combination of theory and practice fosters well-rounded students of creative practice, all of whom are kept very busy throughout the six semesters of their degree. The curriculum is such that they need to hit the ground running from day one. But we've found that not all students are able to fully engage immediately, particularly on the academic side. Frequently, it takes the better part of their first semester just to acclimate to the tertiary environment.

Students enrolled in this polytechnic Creative Industries degree come to their studies from a variety of directions. Some of these include: students directly out of high school; industry up-skillers; secondchance learners; and those who have been out of school for a while and who would now like to begin a career. Most of these people are not accustomed to the consistent intensity that working in a going theatre concern or on a film crew entail, let alone the particular type of thinking that academic research requires.

\subsection{The Certificate}

To this end, we offer a six-month certificate as one of the feeder programmes for the three-year degree. In this certificate, students are introduced to the crafts of acting, theatre technology, and filmmaking. The qualification is designed as a taster for students to gauge whether any of these three disciplines suit them. It is also an excellent diagnostic for academic staff who teach into the degree to gauge whether these students are suited to progressing on to the BPSA.

On the New Zealand Qualifications Framework (the Ministry of Education's guidelines defining all learning levels from secondary through doctoral), the last three years of high school are levels one through three. A Bachelor's degree encompasses levels five, six, and seven. Many high school leavers are able to jump from level three (high school) to level five (bachelors), but there also exists an intermediate step for those who either didn't finish level three in high school or those who, for whatever reason, are not quite ready to enter a Bachelor's programme. On this intermediate step, level four, resides the Certificate referred to herein. Formerly known as the Certificate of Communication and Media Arts, it is now known as the New Zealand Certificate of Study and Career Preparation: Acting, Technical Production and Screen (NZCSCP: ATPS). The cohort varies each semester, with an average of ten students.

The NZCSCP: ATPS consists of four courses, each in two 2-hour blocks (4 hours) per week. The courses are: 1) Identity \& Communication; 2) Collaborative Project; 3) Acting \& Technical Production (ATP); and 4) Moving Images. The courses are designed as a suite that works their muscles from critical theory to hands-on making. Identity \& Communication is the most theoretical; both ATP and Moving Images are hands-on technical/creative craft skill training and practice. This leaves Collaborative Project to cover the nexus between theory and practice. This is where the students learn "21st Century Skills" through project centred learning.

The Collaborative Project course, as delivered in the manner described herein, has gone through two semesters, both in 2019. Previously, the course had a different lecturer who brought his own content to the course descriptor's rather broad learning outcomes. When I inherited this course, I brought together content and approaches from a few similar courses l've developed through my fifteen years of teaching. As the course is shared with another lecturer, the decision was made early on to split the course in half; I would teach the first term, the other lecturer would teach the second term. Collaborative Project thus became two discrete learning experiences. For my half, the challenge was to distill the content I'd previously delivered over a full semester - to Level 6 students - into a short and sharp seven weeks delivered to Level 4 students.

\subsection{Course Philosophy}

Creative students entering an academic environment, but not yet in a Bachelor's programme, need specially tailored skills as part of the "Bachelor's Taster". In addition to the above listed skills and knowledge, a BPSA academic staff identified a gap in their understanding and appreciation for academic research.

Academic research is not merely a practiced activity; engagement with academic research engenders a particular thought process, what Rinatovna calls "Research Thinking" [5]. Skills acquired on the journey through academic research include the ability to clearly focus an inquiry; the ability to construct a framework for investigation and potential resolution of their inquiry; the ability to conduct a focused search for previous research that has been conducted along said line of inquiry; the ability to generate 
informed speculation and to back up that speculation with the research of others who've come before them; and the ability to think critically about any and all information that is sourced. It is an exercise in the systematic acquisition and generation of knowledge, and a way to organise the pursuit of one's natural curiosity. Curiosity as an inherent human quality is the underlying driver at each step of the learning module being discussed here.

Ancillary skills targeted by the course include: how to narrow down ideas from the global to the granular; different approaches to collaboration; active listening; creative negotiation; how to respectfully critique others; tools and techniques for collaborating remotely; and how to develop research into a coherent presentation. Students acquire the confidence and skills to speak compellingly to a group, and at the end of this seven-week module, they have succeeded in presenting their research.

\section{METHOD}

\subsection{Contextualisation}

To help the students connect to the content of the Collaborative Project course, initial contextualisation needs to occur. Students are first asked to discuss their career aspirations - be they acting, backstage work, or filmmaking. They are then led in a guided discussion exploring career pathways in the creative industries, focusing on the increasingly prevalent model of the portfolio career [6], wherein actors, stage technicians and film workers (used as examples for these students) sustain themselves through multiple income streams. A filmmaker, for example, might derive income from working on a film crew (as a casual employee or independent contractor), making corporate videos (as a part-time employee), shooting and editing web-bound commercials for small businesses (as a sole trader/sole proprietor), and writing, producing, directing and shooting their own independent film (requiring film commission or private fundraising). An actor might not only seek and find parts in local theatre, TV and films, but also might found and run a small theatre company of their own, which also requires fundraising. In order to be successful at fundraising for their own projects, a creative practitioner needs to know how to put a proposal together and also how to deliver that proposal as a presentation. Such presentations require research to build a compelling case evidencing that their endeavour fills a gap in the creative landscape.

When explained to the students, the ability to present one's own research is framed as a necessary skillset to succeed in both their academic and their professional creative career. The penny drops for them when they make the connection between research skills and their own creative futures. After that initial discussion (and the occasional revisitation of this idea throughout the seven weeks), the students are (largely) onboard. In that this is not directly related to the craft-skills they seek to acquire, student buy-in is crucial to keep them connected through what will be a new and at times challenging process.

\subsection{Self-identification}

On day 1, the students all sign up on a Roster in Google Sheets, engaging immediately with collaborative cloud-based media. They include their email, phone \# (optionally), and then a number of links which are steadily added to throughout the module. I create the columns; the students add the links to their individual Google Docs and then to their team-built Google Slides.

The students are then tasked with dividing themselves into teams of more or less the same size, and naming their teams. Both activities provide rich opportunities to exercise collaborative muscles. They first must grapple with how they are going to divide themselves; by which method will they decide? As this is their first week in the programme, they are just beginning to get to know one another. Now, they must form sub-groups, deciding whom is grouped with whom. This involves a number of interactive judgement calls and invokes criteria such as: perceived social currency (of both self and others); perceived level of contribution to the team; friendships that have already begun to develop (the course is on Thursday); and general simpatico-ness.

By the time they need to formulate a team name, they've already been through a collaborative experience with one another, have self-identified as a team, and have begun to carve out a language by which to work as a group. Now comes their first creative collaboration which, by its nature, calls on them to activate their imaginations, contribute a name or two, then decide as a group which name to go with. Some teams are more egalitarian, and for others, this is where the pecking order begins to form itself. 
Incidentally, in the past two semesters, the teams have named themselves: "Los Bandidos", "Reverse Oreo", "The Mana Rangers", "Legends", “The O.C.", and "I Don't Like Glue (IDLG)".

\subsection{Process Intro}

For the remainder of the course, they will follow a process in eighteen steps which culminate in their final research presentations. The following enumerates those steps, interspersed with notes about the teaching delivered at various points along the way. The class sessions are numbered in the way they are $(1 a, 1 b, 2 a, 2 b$, etc.) to denote week numbers. Collaborative Project is delivered in two 2-hour sessions each week. In the first semester of 2019, it was on two different days; in the second semester, both two-hour sessions occurred on the same day, one in the morning, the other in the afternoon.

\subsection{The 18-Point System (with Sessions Breakdown and Teaching)}

Within this seven-week module, the students were guided through an 18-step process that culminated with them presenting their research:

\section{Session 1a}

$\Longrightarrow$ Intro to course 1 - Contextual discussion; Course docs; H\&S induction; sign up on G-Sheets Roster;

\section{Session 1b}

$\Rightarrow$ Intro to Course 2 - discussion: What is Collaboration?

$\Rightarrow$ Division into Teams

\section{Session 2a}

$\Rightarrow$ Mapping out of Assignment 1 / discussion

$\Rightarrow$ Over-arching theme: change or transformation / discussion

$\Rightarrow$ (Technique): Brain-writing vs. Brainstorming

Step 1 - Topic Brain-writing - on paper

$$
\text { - Trello• }
$$

$\Rightarrow$ Intro to Trello

Step 2 - Casting a Wide Net for a Team Topic (top 3 from brain-writing, positives \& negatives listed)

\section{Session 2b}

Step 3 - Ideas Critique, Compare, Contrast \& Choose - discussion

Step 4 - Breaking down into Sub-Topics (through another narrowing-down process)

Step 5 - Allocation of Sub-Topics (each team member chooses one which becomes their personal sub-topic research)

\section{Session 3a}

$\Rightarrow$ Introduction to the Research Question

Step 6 - Formulating a Team Research Question - workshop

\section{Session 3b}

Step 7 - Research instructional \& mentored test run

Step 8 - Students Gather research

\section{Session 4a}

- Google Docs •

$\Rightarrow$ Google Docs lesson

Step 9 - Each team member builds their section on G-Docs (shared with teammates), migrates Trello quotes onto G-Doc, and summarises each quote. (continue at home, due next session)

\section{Session 4b}

$\Rightarrow$ Image sourcing lesson (how to use Creative Commons search and attribution) 
Step 10 - Images gathered and compiled onto G-Docs

- Google Slides •

$\Rightarrow$ Presentation Workshop (including G-Slides instructional)

\section{Session 5a}

$\Rightarrow$ Critical Thinking module

Step 11 - Teams prepare quick sharing of research to date and flesh out a rough list of main points.

\section{Session $\mathbf{5 b}$}

Step 12 - Based on their research to date, each group fleshes out their main points to the class, feedback is given, incorporating critical thinking

Step 13 - Students continue to refine their G-Docs prep document (honing summaries, distilling into bullet points, gathering images with attributions), incorporating critical feedback.

\section{Session 6a}

Step 14 - Slide construction [plus any other still-to-be-completed work on their preparatory documents]

\section{Session 6b}

Step 15 - Slides completed, students begin rehearsing

\section{Session 7a}

Step 16 - Full REHEARSAL (formative assessment $w /$ feedback)

Step 17 - Students make adjustments to their presentations based on Rehearsal feedback

\section{Session $7 b$}

Step 18 - FINAL PRESENTATIONS - summative assessment

\subsection{Process Description}

True to the intention of the course, to foster collaborative skills, the students are given broad provocations that they must then work out how to accomplish. The over-arching provocation for their research is that it needs to relate to change or transformation. They are then guided in a discussion aimed at identifying their passion for creative practice and then zeroing in on various aspects of their aspiring practice that are undergoing change (e.g. how social media is changing western culture or how the popularity of film genres has changed over time in relation to cultural and social values).

What follows is an elucidation of Section 3.4:

\subsubsection{Step 1 - Topic Search 1: Brain Writing}

They jump start this process with an activity known as "brain writing", which VanGundy (1984) espouses as an alternative to brainstorming. He contends that in the brainstorming model, the more vocal team members will begin verbalising ideas which result in the team's collective imagination narrowing down to a more limited scope, promoting more of a "groupthink" mentality than fostering individual imagination. Brain writing simply describes each team member silently writing down their ideas and then sharing them later. This can level the idea-generation playing field [7]. The students are encouraged to come up with at least five ideas.

\subsubsection{Lesson: Introduction to Trello}

Trello is an application (both on the web and as an app) that enables the collaborative arrangement of ideas. It has many uses, not the least of which is as a project management tool.

How Trello works: The overall page for each project in Trello is called a "board". On the board are several "lists" in which sit a number of "cards". When a card is clicked on, a lot of other information is revealed. Here, users can add attachments and links of all kinds. They can also communicate with one another using "@" symbols right on the cards, which then push messages out to the relevant member(s). The power of Trello is the ability to move these cards and lists around until an optimal configuration is found. In this sense, Trello is a framework that enables the user to visually sort and organise their ideas. (In fact, I now use Trello for all my course planning, including this one!) 
The students are ushered through the interface with the help of a sample Trello board prepared beforehand by the lecturer. This board is offered as an exemplar for the students to emulate when they later create and populate their own boards.

\subsubsection{Step 2 - Casting a Wide Net for a Team Topic}

The students each choose their top three ideas from the brain writing session and create individual cards for them on the team Trello. They then take a few minutes to write comments on each card their own and their teammates'. Here, they note the upsides (reasons it's a solid and workable idea) and downsides (reasons it's not) of that topic being adopted as the team's topic. The intention here is for the students to articulate in writing their evaluation of each suggested topic in hopes that they might think a little more deeply about their evaluation, and choose their words a little more carefully.

\subsubsection{Step 3 - Ideas Critique, Compare, Contrast \& Choose - discussion}

Once Step 2 is completed (about 20-30 minutes), the students then gather in their teams and have a face-to-face discussion in which they narrow the topic options down to two or three. There is then a few minutes of class time set aside for each team to share the plusses and minuses they'd written (and discussed) for the two or three topic options they've chosen. Any feedback from the rest of the class is welcomed. Out of this process, each team finally commits to their one chosen topic.

\subsubsection{Step 4 - Breaking down into Sub-Topics}

Here, the team narrows down the chosen topic into more granular sub-topics. For example, if the topic were "Comparing \& Contrasting Theatre \& Film", the students might arrive at sub-topics such as: Time (how each platform creates different relationships with time); Movement (actors moving across a stage vs. Actors moving across a frame and camera movement through space).; and Audience Engagement. Students are encouraged to generate more sub-topics than team members so that each member has some choice in which one they choose.

\subsubsection{Step 5 - Allocation of Sub-Topics}

Step 4 flows directly into Step 5 , where the team members decide on who will take each sub-topic. This will be their focus of research for the next five weeks. (We're still in week 2; see 18-step list above).

\subsubsection{Lesson: Introduction to the Research Question}

Using a mixture of YouTube videos and sections of articles read aloud by the class (both used to stimulate discussion), the students are given the core concepts of what a Research Question (RQ) is and is not, as well as the importance of the $R Q$ as a vital element to focus all subsequent research and writing in any given project.

\subsubsection{Step 6 - Formulating a Team Research Question - workshop}

In this 80-minute session in a mentored environment, students develop both a Team RQ (in two iterations) and their individual RQ's (also in two iterations. Each development session (10 minutes) is followed by a brief class discussion section (10 minutes). See below:

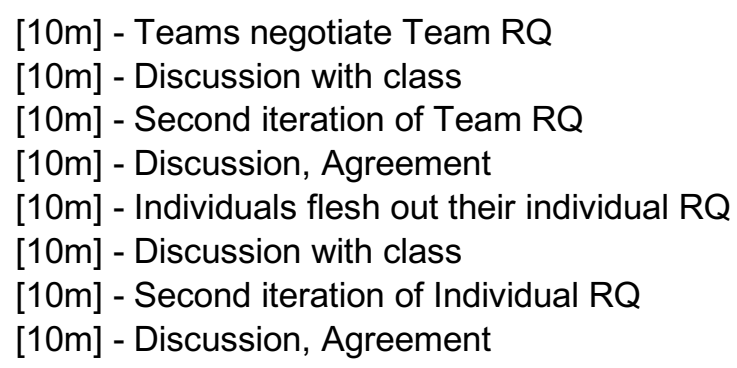

\subsubsection{Discussion Around Formulation of Research Question:}

The motivation behind the relatively short durations of these stages is twofold. First, the intention of this module is to give these certificate students a taster of the research experience; it is not meant to be deep training. As such, the students are being taken on a whistle-stop tour of the high points of the journey. Secondly, l've experimented over the years with varying the degrees of time and depth in introducing new concepts (such as the RQ) to a variety of student cohorts. What seems to work best 
(at least for the Performing \& Screen Arts students here at Unitec) is to let them dip their toes quickly into the water and then out again by engaging in a rapid-fire journey through the material. This highenergy experience provides an association of excitement with the content, and also affords them a perspective on the entire process within one attention span cycle. After the rapid-fire session, and after they've had a chance to recover and to think about it, subsequent sessions are planned in which we then go much more deeply into the subject. However, as this is just a seven-week module for prebachelor students, the entire process is necessarily much more abbreviated. Furthermore, students were given the freedom to change their RQ (as is par for the course in the "real" world), so they knew they weren't locked in by the outcome of this very quick session; it was just assembled in this way to give them an overview.

Common sense and logical procedure would normally see the team RQ being formulated just after the groups chose their team topics and just before they divide their work into sub-topics. Indeed, the team $R Q$ would serve better to inform the student researchers what the sub-topics should be. Admittedly, to choose the topic, the sub-topics and then search for a RQ is not the ideal order. However, it was felt that the introduction of the smaller learning units within the semester would be more effective if clustered and not broken up as much. The idea here is to chunk the learning elements into like units. Hence what would ideally have been:

- Determination of Team Topic

- RQ lesson/workshop

- Determination of Individual Sub-topics

- How to Research

- Student Research into Sub-topic

was instead delivered as:

- Determination of Team Topic

- Determination of Individual Sub-topics

followed by

- $\mathrm{RQ}$ lesson/workshop

- How to Research

- Student Research into Sub-topic

It was a judgement call based on the necessary efficiencies of the tight schedule.

\subsubsection{Step 7 - Research Instructional \& mentored test run}

Students are shown resources for finding already-existing research. These include the Unitec Library Website plus Google Scholar (logged into the school's network to enable access to subscription-based archives) and, of course, plain Google web. The Unitec website was given the most time, ushering students through the various directories. Discussion of primary/secondary sources was followed by the admonition to not cite Wikipedia as a source. A discussion of plagiarism ensued with the students engaging with a gamified plagiarism quiz. Finally, a discussion about how to read the abstract, the introduction and the summary of an article in order to get the gist of the content, which would help them determine if they wanted to plunge into the entire piece.

Additional sources include online newspaper and magazine articles.

Finally, the students were instructed to attach links inside their Trello sub-topic page to the articles they wanted to use. This centralised the entire process up to this point on their team's Trello board.

\subsubsection{Step 8 - Students Gather Research}

This is the step where the students, in class, conduct their research into their sub-topic.

\subsubsection{Lesson: Google Docs}

The students are given a short introduction to Google Docs as a live collaborative platform. In the first iteration of this course, I had each team create a Google Doc for their shared research. This proved unwieldy and impracticable because several people were contributing to the document, causing text to jump around. Also, each page had all the sub-topics, so focusing on any one sub-topic became laborious at best. So, in the second iteration, the students each created their own Google Doc for their own research. They shared it with "anyone with the link", shortened the URL's with Bitly, and shared the link on the Roster (see "Self-Identification" below). This allowed the students (and me) to see what 
each of the other team members were doing - but each in their own virtual space.

3.5.13 Step 9 - Each team member builds their section on G-Docs (shared with teammates), migrates Trello quotes onto G-Doc, and summarises each quote.

Once the students had gathered their individual research into their Trello cards, they were instructed to read through each article and to cut and paste the sections they wanted to use right into their Google Doc. Underneath each pasted section, they were to paste the URL from whence the article came. Underneath that, they were guided to summarise, in their own words, the essence of the pasted section ("as if you're telling someone a story about it") without using any words or phrases from the original material. Finally, they were to read the summary they'd just written and distill that into a few bullet points (with no more than five words each). In that they did this for each section of each article that they had sourced, the students were encouraged to only pull the best three or four extracts from the articles.

\subsubsection{Lesson: Sourcing Images}

This was a quick tour through the (new as of April, 2019) Creative Commons (CC) search engine, along with some videos from $\mathrm{CC}$ extolling the virtues of pulling from, and contributing to, the Creative Commons. I supplemented these with a facilitated discussion on the importance of always attributing image sources. Finally, the students were given a step-by-step procedure for attaching the attribution to the image itself.

\subsubsection{Step 10 - Images gathered and compiled onto G-Docs}

The students found images that either directly or metaphorically illustrated the distilled research they had just created on their Google Doc. Now, they are adding images to each major idea - putting them right into Google Docs, just under their text, effectively now using their Google Doc as a worksheet on which their presentations are being constructed.

\subsubsection{Lesson: Presentation Workshop (including Google Slides instructional)}

This is a fun introduction to presentation styles and techniques wherein the students are given a quick tour through the work of a number of experts in presentation, body language, powerful speaking and other related subjects. They were then shown video excerpts of three presentation styles: Bill Gates introducing Windows Vista; Steve Jobs introducing the (first) iPhone; and Dick Hardt discussing new authentication protocols, which he called "Identity 2.0". Each of these presentations are delivered in very different styles. Following this, the students broke themselves into teams of three and had 20 minutes to create a presentation on that day's breakfast, lunch, and dinner - one in each of the above styles. The class had to guess in whose style each was presenting. This got the students quickly pulling images into a presentation, then safely inhabiting the roles of other people while they took their first steps presenting. Most students enjoyed exaggerating the characters of these three speakers and it helped defuse their nervousness about public speaking.

\subsubsection{Lesson: Critical Thinking}

After watching a couple of excellent YouTube videos on the subject, students are introduced to various pages on the website of The Foundation for Critical Thinking as well as Bertrand Russell's 10 Essential Rules for Critical Thinking. The 10 rules are spread evenly across student pairs who discuss among themselves then share with the class examples of how each rule has been, or may be, applied in their lives.

3.5.18 Step 11 - Teams prepare quick sharing of research to date and flesh out a rough list of main points.

This occurs within each team. They're preparing to informally share with the class the main points of their research and how they intend to focus it in their presentations. The rest of the class listens

3.5.19 Step 12 - Based on their research to date, each group fleshes out their main points to the class, feedback is given, incorporating critical thinking.

Step 11 is the preparation for Step 12. Here, the students share their research with the rest of the class, who listens actively and offers feedback to each group, weaving critical thinking into their feedback. 
3.5.20 Step 13 - Students continue to refine their G-Docs prep document (honing summaries, distilling into bullet points, gathering images with attributions), incorporating critical feedback.

This is done within each team.

3.5.21 Step 14 - Slide construction [plus any other still-to-be-completed work on their preparatory documents]

Students port their images plus any text (either as part of their slide or as part of their presenter notes) onto Google Slides. Each team has allocated a certain number of slides per team member, but this, of course can be added to or deleted from. This is an intense studio-style session.

\subsubsection{Step 15 - Slides completed, students begin rehearsing}

(Self-explanatory)

\subsubsection{Step 16 - Full REHEARSAL (formative assessment $w /$ feedback)}

Students go through their entire presentations. Each one is followed by a feedback session with comments from both the lecturer and the other students.

\subsubsection{Step 17 - Students make adjustments to their presentations based on Rehearsal feedback.}

The teams discuss which feedback they want to/need to/have the time to take on, then beaver away at those changes to perfect their presentation in time for delivery.

\subsubsection{Step 18 - FINAL PRESENTATIONS - summative assessment}

These are recorded on video for fair marking and moderation.

\subsection{Excerpts from Student Reflections}

\subsubsection{Student 1}

After finishing this project, I realise how much closer we got throughout the project and how much more we began to work with each other and bounce ideas off each other.

\subsection{Student 2}

I was affected by learning new things that I didn't know were in front of me, for example learning how to reference and put your own words into information that you found. I also learnt how to use google slides which will help do presentations more easily in the future.

\subsubsection{Student 3}

What l've learnt that can be applied to future projects is definitely networking, but also learning how to reference images that you use and to also not reference Wikipedia as well. Coming together to work as a team, figuring out solutions and coming up with a project and also researching but also making connections [...] this would come in handy in the future when needed or at any time.

\subsubsection{Student 4}

During our collaborative project, I learned about where I need to grow, all the factors of a project, and what skills are needed for collaboration. For our project we made a slideshow about how film genres change with cultural values, each of us looking at a different genre.

We were shown how to obtain images lawfully using the creative commons. Its limited range meant having to think creatively, which is better than having infinite choice in my opinion. These are useful tools that I will use with every given chance in the future.

\subsubsection{Student 5}

I found working on this collaborative project interesting as I got to work both individually (individual research), and in a team to create a slideshow presenting points for a topic creatively. It was also interesting to see the process of developing the presentation, watching it progress from start to finish.

Each step of the process helped build up the result of the presentation. We started by breaking it down, from listing possible topic ideas to narrowing it down to a final idea and then researching and finally, 
creating the slides and presenting it. I contributed what I could towards each step to help my group so that there was equal contribution among everybody, and nobody ends up doing most of the work on their own.

\subsection{Student 6}

In general, the method was extremely helpful for me. I will certainly go through this method again when presentation projects like this are to be done.

\subsubsection{Student 7}

During my experience working on this project I learnt that it is not all so easy joining ideas with others straightaway. I learnt how to have more patience for others as well as the strategy to join everyone's ideas in a tidy more structured way. Working while having a time limit is always difficult especially working on a project with a lot of different pieces connected to it. Trello is an easy straightforward application that can collect all your data in a tidy form. I really enjoyed being introduced to [it] and will for sure be using it in the future. While participating in this project I feel as if I struggled in the area of time management. Personally, I feel if I was to be more organised and focused in my time of creating my google slides I would have been less stressed and more confident about my work. Running through the presentation in the end I felt relieved. Not because of the fact the task was now over but because I personally feel my work that I had completed was more structured and organised then I originally thought.

I enjoyed working on this presentation and can positively say that it has definitely taught me strategies that will be helpful in my future. I also enjoyed working in my team that I was a part of, I feel as if this project brought us closer and it is also nice to see how others work compared to how I personally do myself. I would say we all learnt at least 1 new thing from the help of each other.

\subsubsection{Comments on Student Reflections}

A predominant theme in the students' reflections was the value they derived from collaborating with others in a team effort, embodying Lopatto's assertion about the importance of social relationships in the development of an undergraduate research culture [2]. Students also learned the the importance of time management in working on complex projects. The use of tools for research and collaboration emerged for them as a key skill for both gathering and organising information. The students had felt challenged by the project early on, but upon reflection, experienced themselves as having engaged with it successfully.

\section{CONCLUSION}

The 18-step model described above is, admittedly, a very light-touch and rapid-fire introduction to the basics of creating a research presentation. Its limitations, due largely to its very short time frame, can also be seen as its strengths. The lack of time to go into depth is counterbalanced by the process overview students gain by sprinting through the fundamental elements. Further time can and should be spent inside the Bachelor's degree to drill down to the finer details that proper engagement with the research process requires. However, for pre-Bachelors' students who are mainly focused on acting and filmmaking, a short foray into the research world has shown to provide a worthwhile entrée into this arena. Further exploration into techniques for warming pre-Bachelor students to the value and enjoyment of research could reveal a more optimal structure and methodology.

\section{REFERENCES}

[1] Lanza J. Whys and Hows of Undergraduate Research. Biosci Oxf. 1988 Feb;38(2):110.

[2] Lopatto D. Undergraduate Research as a High-Impact Student Experience. Peer Rev. 2010 Spring;12(2):27-30.

[3] Bowers J, Parameswaran A. Differentiating undergraduate research. Teach High Educ. 2013;18(5):453464.

[4] Musante S. Learning How to Ask Research Questions. Biosci Oxf. 2010 Apr;60(4):266.

[5] Rinatovna KL. Psychological and Pedagogical Foundations of Undergraduates' Research Thinking Development Process. Procedia - Soc Behav Sci. 2017 Feb 21;237:1405-11. 
[6] Wilson C. The rise of the portfolio career in the creative industries [Internet]. Culture 360. 2015 [cited 2015 Jun 7]. Available from: http://culture360.asef.org/magazine/the-rise-of-the-portfolio-career-inthe-creative-industries/

[7] VanGundy AB. Brain Writing for New Product Ideas: An Alternative to Brainstorming. J Consum Mark. 1984 Jan 1;1(2):67-74. 